\title{
Repetition Blindness and Aging: Evidence for a Binding Deficit Involving a Single, Theoretically Specified Connection
}

\author{
Donald G. MacKay, Michelle D. Miller, and Sarah P. Schuster
}

\begin{abstract}
This study tested 2 main hypotheses for explaining repetition blindness (RB), a difficulty in encoding and recalling rapidly presented repeated words in sentences. Under 1 hypothesis, RB reflects an inhibitory process and should be more pronounced in young than in older Ss, who typically exhibit diminished inhibitory processes. Under the second hypothesis, $R B$ reflects a failure to bind a specific connection: The second connection from the single node for encoding a repeated word is difficult to form under time pressure. Under this binding hypothesis, young adults should exhibit less RB than older adults, who typically require more time to form new connections. Results supported a version of the binding hypothesis but contradicted the inhibition hypothesis, and did not support hypotheses whereby RB reflects either a refractory effect or perceptual fusion of the repeated words.
\end{abstract}

Repetition blindness (RB) refers to the reduced probability of encoding and recalling a word or letter because of prior occurrence of the same word (or letter) in a rapidly presented list or sentence (see, e.g., Hochhaus \& Marohn, 1991; Kanwisher, 1987, 1991; Kanwisher \& Potter, 1989, 1990; MacKay, 1969). For example, when the sentence "They wanted to play sports but sports were not allowed" is presented at $150 \mathrm{~ms}$ per word in a rapid serial visual presentation (RSVP) task, the second occurrence of sports is more difficult to recall than the same word sports in the nonrepeating context "They wanted to play ball but sports were not allowed" (Kanwisher, 1987).

Encoding of single, theoretically specified connections is the main focus of this research, which represents an advance over previous work in which encoding processes often involved hundreds of unspecified connections. This research is also the first to compare RB in young versus older adults. This comparison is relevant to understanding the basic mechanism that underlies $\mathrm{RB}$ and enables a test of predictions derived from four welldocumented classes of related phenomena in the field of cognitive aging, which are discussed next.

\section{Inhibition Deficits and RB}

The phrase inhibition deficits refers to the reduced effectiveness of inhibitory processes in older adults (see, e.g., Birren \& Woodruff, 1983; Connelly, Hasher, \& Zacks, 1991; Hasher, Stol-

Donald G. MacKay, Michelle D. Miller, and Sarah P. Schuster, Psychology Department, University of California, Los Angeles.

Support from National Institute for Aging Grant R01 AG 09755 to Donald G. MacKay and National Science Foundation Graduate Fellowship to Michelle D. Miller is gratefully acknowledged. We thank Deborah Burke for helpful comments on an earlier version of this article, Lise Abrams for assistance in computer programming, and the Pomona College Psychology Department for generously lending GenPrime and other equipment essential for this study.

Correspondence concerning this article should be addressed to Donald G. MacKay, Psychology Department, University of California, Los Angeles, California 90024-1563. fus, Zacks, \& Rympa, 1991; see Hasher \& Zacks, 1988; McDowd, Oseas-Kreger, \& Filion, 1994, for reviews) and are relevant to a major framework that has been advanced to explain RB. According to this framework, an inhibitory process that follows activation of lexical and phonological nodes may reduce detectability of the second instance of a rapidly presented word (see Hochhaus \& Marohn, 1991, for detailed theories of $\mathrm{RB}$ within the inhibition framework). Of course, inhibitory processes have never been studied in older adults for the rapid processing rates required to induce $R B$. However, if $R B$ reflects inhibitory processes that resemble those examined in current paradigms, then young adults should exhibit more RB than older adults.

\section{RB and the Refractory Period}

Refractory periods (the time during which a response to a stimulus is slowed by occurrence of a previous response to the stimulus) are much longer for older adults than for young adults. In older adults, reaction time to a signal that occurs simultaneously with onset of the response to a previous signal is $38.8 \%$ longer than to an identical signal that follows $2,000 \mathrm{~ms}$ after offset of the previous response; in young adults, such a reaction time is only $15.5 \%$ longer (Welford, 1977). Thus, if RB is related to refractory period effects, then RB should interact with age, repetition, and presentation rate, and older adults should experience more $\mathrm{RB}$ than young adults at moderate rates, but equivalent RB at fast and slow rates. To illustrate, assume that the probability of recalling an unrepeated word varies between 0 and 1 as a function of presentation time in the same way for young and older adults. For very fast rates that fall within the refractory periods of both young and older subjects, RB will be maximal and age-independent, and for very slow rates falling outside these refractory periods, recall functions for repeated and unrepeated targets will be identical, with no RB for young or older subjects. However, a shift from maximal to minimal RB will occur at some intervening or moderately fast rate, and the moderate rate at which this refractory shift occurs will be faster for young than for older subjects, because refractory periods are shorter for young than for older subjects. These age differences 
predict Age $\times$ Presentation Rate and Repetition $\times$ Age $\times$ Presentation Rate interactions. Moreover, the recall function for repeated targets should vary as a cubic function of rate and should deviate from the recall function for unrepeated targets at a faster rate for young than for older subjects. Equivalently, if recall of unrepeated targets varies as a linear function of rate, recall functions for repeated targets will exhibit nonlinearity at a faster rate for young than for older subjects because of young subjects' shorter refractory periods.

\section{RB and Perceptual Fusion}

Fine and Reeves (1991; see also Humphreys, Besner, \& Quinlan, 1988) developed a perceptual fusion account, whereby RB occurs when subjects process two words that are identical in meaning within the same hypothetical time window, so that repeated words fuse together and are represented as one in the subject's mind even when they differ in physical characteristics, for example, upper versus lower case (Kanwisher, 1987) and visual format (e.g., nine vs. 9; Bavelier \& Potter, 1992). MacKay and Miller (1994) recently produced evidence that removed a major obstacle to this fusion account of RB by unambiguously demonstrating semantic blindness, the occurrence of $\mathrm{RB}$ at a semantic level rather than at sensory, phonological, or orthographic levels. Moreover, when combined with Kline's (1984) trace persistence hypothesis, a perceptual fusion account predicts interesting effects of age on RB. Under this trace persistence hypothesis, stimulus traces persist longer in the nervous system of older adults, causing a wide range of age effects on perceptual fusion. Thus, critical flicker-fusion frequency (CFF), the minimal on-off rate required for a subject to perceive a flickering light as fused or steady-state, is lower for older than for young adults. If the CFF for a given light is 33 cycles per second (cps) for 60-year-olds, then $40 \mathrm{cps}$ or more are required for 20-year-olds to perceive the same light as fused (Botwinick, 1984 , p. 208). Similarly, negative afterimages remain visible longer for older than for young adults (Kline \& Nestor, 1977), and color fusion exhibits longer time characteristics in older adults: When two lights of complementary wavelengths are presented sequentially, for example, red and then green, older adults experience color fusion (i.e., they see yellow) with longer interstimulus intervals, as though the trace of the first color persists longer for them than for young adults (Kline, Ikeda, \& Schieber, 1982). Finally, if the left half of each letter of a word is presented briefly in one time frame, followed by the right half of each letter in a subsequent frame, older subjects can identify the word at longer interstimulus intervals than can younger subjects, which again suggests that the trace of the first stimulus persists longer for older than for young adults (Kline \& OrmeRogers, 1978; see also Di Lollo, Arnett, \& Kruk, 1982).

According to Kline (1984), age-linked trace persistence occurs at all levels and may even account for general slowing, which is the fact that older subjects typically respond more slowly than do young subjects in a wide range of psychological tasks (see, e.g., Birren, Woods, \& Williams, 1980). Thus, if RB reflects a semantic-level trace persistence effect with shorter intervals required for fusing repeated targets in young subjects, age, $\mathrm{RB}$, and presentation rate should interact in the same way as for the refractory period hypothesis.

\section{Binding Deficits and RB}

Binding, or new learning, deficits refer to the reduced ability of older adults to form new connections (see, e.g., MacKay \& Burke, 1990, for a review) and are relevant to the hypothesis that $\mathrm{RB}$ reflects a difficulty in encoding repeated words: Encoding a repeated word requires two instances of connection formation from the same node, so the second instance of a word repeated closely in time becomes more difficult to bind or encode than an unrepeated word (for theories incorporating this encoding assumption, see MacKay \& Miller, 1992, 1994; Wickelgren, 1965). Again, binding processes have never been studied in older adults using the extremely rapid processing rates required to induce RB. However, if RB reflects binding processes that resemble those examined at slower processing rates, older adults should exhibit greater RB than do young adults. With respect to effects of rate on $R B$, predictions from different binding theories are less consistent. However, one binding theory (MacKay \& Miller, 1992, 1994), described in detail in the Discussion section, predicts that RB will decrease systematically as a function of rate for both young and older subjects.

In summary, the present study tested the following predictions: that young adults will exhibit more RB than older adults (inhibition deficit hypothesis); that older adults will exhibit more RB than young adults (binding deficit hypothesis); that $R B$ will interact with presentation rate, with greatest $R B$ at the fastest presentation rates (refractory period and fusion hypotheses); that $R B$ will vary as a cubic function of age and presentation rate, with equivalent $\mathrm{RB}$ at the fastest rates, greater $\mathrm{RB}$ for older than for young adults at moderate rates, and no RB for young or older adults at the slowest rates (refractory period and fusion hypotheses); and that recall of repeated targets will vary as a nonlinear function of rate, with the nonlinearity occurring at a faster rate for young than for older subjects (refractory period and fusion hypotheses). In addition, linear trend analyses will test the background assumption of the refractory period and fusion hypotheses, which is that recall of unrepeated targets varies with presentation time in the same way for young and older adults.

\section{General Methodological Issues}

Our procedure was originally developed by Kanwisher (1987). Sentences were presented using the RSVP technique, and immediate verbatim recall was compared for identical target words in contexts that differed by a single word. The prior, or pretarget, word was the same for repeated target sentences but different for unrepeated target sentences. For example, recall of the word work was compared in "It was time to score so work had to be done" (unrepeated target version, target and pretarget italicized) versus "It was time to work so work had to be done" (repeated target version). Thus, a subject experiencing RB might report the repeated target sentence as "It was time to work so had to be done." Curiously, no other study has examined effects of presentation rate on RB. Kanwisher systematically manipulated presentation rate for RSVP lists that all contained repeated words, but her subjects' task was to report which word was repeated. The relative retrievability of repeated versus unrepeated targets, which is essential for specifying $R B$, was undetermined. 
Target retrievability can be measured in two ways, which we call the traditional and conditional measures. Each measure has strengths and weaknesses, and full analyses of both measures are needed to provide an appropriate database for testing our predictions (see Brown \& McNeill, 1966, on the use of complementary measures under such conditions). The traditional measure reflects how often subjects correctly recall a target word, with RB calculated as the percentage correct target report for unrepeated minus repeated targets. This traditional measure has two weaknesses. One concerns trials in which subjects recall only the target or the pretarget, but not both, and the remaining recalled words do not specify whether the target or the (identical) pretarget was recalled. These trials are scored as correct in the traditional measure, but this procedure works against observing RB and may be unduly conservative. A second weakness is that the traditional measure includes trials in which subjects do not attend to, perceive, or recall the pretarget. Because facilitation rather than inhibition of repeated targets can be expected for these trials on both theoretical grounds (MacKay, 1990) and empirical grounds (Kanwisher, 1991, Experiment 5), the traditional measure may again underestimate the true degree of RB.

The conditional measure overcomes both weaknesses of the traditional measure by counting target recall as correct only if the pretarget is also included in recall, a clear indication of pretarget perception. By discarding trials in which the pretarget is unrecalled and perhaps unperceived, the conditional measure therefore provides a more suitable descriptive estimate of $\mathrm{RB}$ as a theoretical construct. However, the conditional measure also has a weakness that is directly relevant to studies of cognitive aging: Because young subjects tend to recall the pretarget more often than do older subjects, the conditional measure discards relatively more data for older than for young subjects, which presents a problem for standard statistical analyses. To deal with the costs and benefits of both measures, we follow the general prescription of Brown and McNeill (1966) for such problems - to report the data fully and to analyze them in more than one way. ${ }^{1}$

Ceiling and floor effects are a special problem for studies of RB. When presentation rate is varied across a wide range of rates, recall will in principle hit ceiling at a faster rate for unrepeated than for repeated targets, so that a Rate $\times R B$ interaction is inevitable and perhaps uninteresting. Moreover, with the same range of rates for young and older subjects, recall will in principle hit ceiling at a faster rate for young than for older subjects, so that a Rate $\times \mathrm{RB} \times$ Age interaction is also inevitable and uninteresting. In short, to ensure that the Age $\times$ Rate and Repetition $\times$ Age $\times$ Rate interactions that are predicted by the refractory period and fusion hypotheses are not artifactual, a range of presentation rates must be chosen that avoids ceiling effects for both young and older subjects. This raises the additional issue of how to choose a nonarbitrary level of recall that can be accepted as a ceiling. To solve this interrelated set of floor and ceiling problems, we chose a range of rates for which recall of unrepeated targets was comparable for young and older subjects and was close to $80 \%$ using the conditional measure. This solution avoids differential ceiling effects for unrepeated targets and allows a wide range of possible RB effects for young and older subjects without introducing floor effects that could dis-
Table 1

Subject Characteristics

\begin{tabular}{lrrrrrr}
\hline & \multicolumn{2}{c}{ Young } & & \multicolumn{2}{c}{ Old } \\
\cline { 2 - 3 } \cline { 6 - 7 } \multicolumn{1}{c}{ Characteristic } & $M$ & $S D$ & & $M$ & $S D$ \\
\hline Age $^{\mathrm{a}}$ & 22.0 & 3.3 & & 72.6 & 5.0 \\
Education $^{\mathrm{b}}$ & 15.3 & 1.5 & & 15.6 & 2.4 \\
WAIS Vocabulary $^{\mathrm{a}}$ & 24.2 & 6.8 & & 31.4 & 7.3 \\
Forward Digit Span $^{\mathrm{a}}$ & 7.5 & 1.0 & & 6.7 & 1.5 \\
Backward Digit Span & 5.5 & 1.4 & & 4.9 & 1.2 \\
Health rating & 8.3 & 1.1 & & 8.2 & 1.7 \\
\hline
\end{tabular}

Note. $\quad$ WAIS $=$ Wechsler Adult Intelligence Scale.

${ }^{a}$ Differences between young and older subjects significant beyond $p<$ .05 . ${ }^{b}$ Education in years.

tort the degree of $\mathrm{RB}$ differentially for one group relative to the other.

Method

\section{Subjects}

Participants were 32 young adults (16 men, 16 women; 27 right handed, 5 left handed) and 32 older adults ( 21 men, 11 women, 28 right handed, 4 left handed). None had participated in MacKay and Miller's (1992) study of repetition deafness and aging. All reported normal (or corrected-to-normal) vision and could produce and comprehend spoken English fluently. All but 2 young and 2 older subjects reported English as their native language, and these 4 people rated their English fluency as very high ( $M=4.75$ on a 5-point scale). Table 1 presents background information for each group. Older subjects lived in the community and were highly educated, with about the same mean number of years of education as young adults, all of whom were undergraduates of the University of California, Los Angeles (UCLA). The Wechsler Adult Intelligence Scale-Revised (Wechsler, 1981) Vocabulary subtest and Forward Digit Span showed a commonly observed age pattern: Older adults scored significantly higher on the Vocabulary measure than did young adults, $F(1,62)=223.41, p<.001$, but significantly lower on Digit Span, $F(1,62)=6.39, p<.05$.

All but 1 of the 32 young subjects were paid $\$ 7$ for their participation in the experiment; 1 participated in return for partial course credit in an introductory psychology class. The 32 older subjects were paid $\$ 10$ as members of the UCLA Memory and Cognition subject panel. Half of the older subjects were recruited from the Plato Society, a continuing education program at UCLA, and half were recruited through notices in community centers in the west Los Angeles area.

\section{Materials}

We constructed 16 sentences containing 6 to 12 words $(M=9.5$ words). Each sentence came in two versions that differed only in their pretarget word, pretargets and targets were identical in the repeated target condition and different in the unrepeated target condition (see

\footnotetext{
${ }^{1}$ We do not recommend the joint measure of RB, a third measure in which target recall is scored as incorrect, unless both the target and pretarget are included in recall. The joint measure suffers from the same weakness as the conditional measure and introduces an additional problem that applies especially to studies of RB and aging-floor effects that differentially distort the degree of $\mathrm{RB}$ for older subjects relative to young subjects.
} 
Table 2 for examples). Repeated and unrepeated target versions were similar in meaning and syntax and were always grammatically acceptable. Targets and pretargets were closely matched for average length ( $M$ $=3.5$ letters) and frequency according to Francis and Kucera (1982) and were separated by either one intervening word, as in "People who like books like magazines as well," or two intervening words, as in "The trees were covered with red pears and red apples." Neither the target nor the pretarget occurred at the beginning or end of a sentence or in more than one sentence in the materials.

Materials also included 32 distractors or fillers, half of which were normal sentences with no repeated words, thereby ensuring that subjects did not focus on factors related to repetition. Remaining fillers ( $n$ $=16$ ) were anomalous, formed by omitting one or two words in an otherwise normal sentence (e.g., "When we went to the it was very crowded" instead of "When we went to the store it was very crowded"; see Table 2). Anomalous fillers were included to ensure that subjects could sometimes expect ungrammatical or incomplete sentences, a common consequence of $\mathrm{RB}$.

\section{Procedure}

Subjects were told that they would see sequences of words superimposed one after another in the center of the screen before them and that they were to immediately say each sequence aloud as soon as it ended. They were warned that the sequences would be speeded up by various degrees and that some might seem strange or ungrammatical, but that they should try to recall each string verbatim without "fixing it up" or paraphrasing. Subject output was tape recorded, but was also transcribed "on line" onto scoring sheets by the experimenter (Sarah P. Schuster). A Macintosh Plus computer presented the sequences using a general purpose program (GenPrime) that had been developed at Pomona College (Banks, Burke, Krajicek, \& Whetstone, 1990). A 200-ms warning alerted subjects to the next sequence, which began $120 \mathrm{~ms}$ later. A string of question marks (??????) followed the last word in a sequence, signaling that subjects were to recall the sequence orally. After generating their responses, subjects pressed the space bar to begin the next trial.

Materials were presented at four different rates, three of which were identical for young and older adults: 70,90 , and $110 \mathrm{~ms}$ per word. The fourth rate was $50 \mathrm{~ms}$ per word for young subjects and $200 \mathrm{~ms}$ per word for older subjects. These rates were chosen on the basis of pilot data to represent the best possible solution to the methodological issues discussed in the introduction. In addition, the pilot data indicated that our range of 50 to $200 \mathrm{~ms}$ per word included rates for which $\mathrm{RB}$ is maximal in both groups, another important consideration if the rates for maximal RB differ for young versus older subjects.

Each subject saw 16 fillers and 16 experimental sentences, that is, 8

Table 2

Example Materials

Example sentences, repeated-target versions:

"He is the one who one day will be famous."

"We buy fruit when fruit is in season."

Example sentences, unrepeated-target versions:

"He is a man who one day will be famous."

"We buy juice when fruit is in season."

Nonanomalous filler sentence:

"Good teachers are always patient and kind."

Anomalous filler sentence:

"Good teachers are always and kind."

Note.

Target words are in italics. repeated target sentences plus unrepeated target versions of the remaining 8 sentences, with versions counterbalanced across subjects. Presentation rate varied orthogonally with sentence type, giving 2 repeated and 2 unrepeated target sentences and 4 fillers at each of the four rates. Stimulus order varied randomly across subjects, but average serial position for repeated and unrepeated versions was held constant. Each session began with 4 practice sentences: a repeated target sentence, an un. repeated target sentence, an anomalous filler, and a nonanomalous filler. Practice sentences were presented at the four different rates, in random order except that fastest or slowest rates never occurred first.

\section{Results}

The traditional (percentage correct) measure is shown in Table 3 for repeated and unrepeated targets as a function of presentation rate together with $R B$, calculated by subject as the correct target report for unrepeated minus repeated targets and translated into percentages. As one can see in Table 3, young and older subjects recalled unrepeated targets at comparable levels across all available rates ( $M=85 \%$ vs. $80 \%$ correct). Two analyses of variance (ANOVAs) were carried out on these traditional data, with Age, Repetition, and Rate as factors. The first ANOVA followed a $2 \times 2 \times 4$ design that included all four rates for young and older subjects-three identical rates and two nonoverlapping rates (see Table 3). This ANOVA revealed significant effects for Rate, $F(3,186)=11.69, M S_{\mathrm{e}}=0.356, p<.001$, Repetition, $F(1,62)=49.38, M S_{\mathrm{e}}=0.306, p<.001$, and a Repetition $\times$ Presentation Rate interaction, $F(3,186)=3.91$, $M S_{\mathrm{e}}=0.351, p<.01$. The trend analysis for the relation between Rate and $\mathrm{RB}$ (correct target report for unrepeated minus repeated targets) indicated that $\mathrm{RB}$ decreased as a linear function of Rate, $F(1,62)=13.49, p<.001$, with no cubic trend, $F(1,62)=2.58, p=.14$. Separate trend analyses for relations between Rate and recall of repeated and unrepeated targets indicated significant linear trends for both, $F(1,62)=39.74, p<$ .0001 , and $F(1,62)=5.31, p<.03$, but no significant cubic trends, $F(1,62)=0.28, p=.60$, and $F(1,62)=3.16, p=.08$. Separate trend analyses for young and older subjects recalling unrepeated targets indicated an identical pattern of linear trends for both groups.

Turning to age effects, correct target reports across our range of presentation rates were significantly less likely for older than for younger subjects, $F(1,62)=12.58, M S_{\mathbf{e}}=0.761, p<.001$. This age effect was entirely attributable to repeated targets: Older subjects recalled repeated targets much less often than did younger subjects $(M=54 \%$ vs. $76 \%)$ and exhibited more RB than young subjects averaged across all presentation rates $(M=26 \%$ vs. $8 \%$ ), an Age $\times$ Repetition interaction that was significant at $p<.001, F(1,62)=13.49, M S_{\mathrm{e}}=0.306$. Neither the Age $\times$ Rate interaction, $F(3,186)=1.00, M S_{\mathrm{e}}=0.356, p>$ .39 , nor the Age $\times$ Rate $\times$ Repetition interaction, $F(3,186)$ $=1.37, M S_{\mathrm{e}}=0.351, p>.25$, was significant. A conservative estimate of power to detect a moderate size effect $(f=.25$; Cohen, 1988 , p. 355) at $p<.05$ in these interactions was .84 (Cohen, 1988, Table 8.3.14, p. 316).

Our second ANOVA followed a 2 (Age) $\times 2$ (Repetition) $\times 3$ (Rate) design that included data for only the three rates shared by young and older subjects ( 70,90 , and $110 \mathrm{~ms}$ per word), but gave identical results: The main effect of Rate, $F(2,124)=3.90$, $p<.02, M S_{\mathrm{e}}=0.320$, and the Age $\times$ Repetition interaction, 
Table 3

Mean Correct Target Report as a Function of Repetition Condition and Rate for Young and Older Subjects

\begin{tabular}{|c|c|c|c|c|c|c|c|c|c|c|}
\hline \multirow[b]{3}{*}{ Subjects } & \multicolumn{10}{|c|}{ Rate of presentation } \\
\hline & \multicolumn{2}{|c|}{50} & \multicolumn{2}{|c|}{70} & \multicolumn{2}{|c|}{90} & \multicolumn{2}{|c|}{110} & \multicolumn{2}{|c|}{200} \\
\hline & CTR & $S D$ & CTR & $S D$ & CTR & $S D$ & CTR & $S D$ & CTR & $S D$ \\
\hline \multicolumn{11}{|l|}{ Young } \\
\hline Unrepeated & 77 & 36 & 86 & 26 & 86 & 29 & 89 & 21 & & \\
\hline Repeated & 66 & 37 & 73 & 28 & 78 & 28 & 88 & 25 & & \\
\hline Repetition blindness & 11 & 42 & 13 & 31 & 8 & 40 & 2 & 35 & & \\
\hline \multicolumn{11}{|l|}{ Older } \\
\hline Unrepeated & & & 73 & 30 & 83 & 35 & 74 & 28 & 88 & 25 \\
\hline Repeated & & & 41 & 35 & 40 & 42 & 59 & 37 & 75 & 34 \\
\hline Repetition blindness & & & 31 & 47 & 47 & 55 & 16 & 41 & 11 & 33 \\
\hline
\end{tabular}

Note. Numbers represent percentages. CTR $=$ correct target report.

$F(1,62)=22.43, p<.0001, M S_{\mathrm{e}}=0.246$, were significant, but neither the Age $\times$ Rate $\times$ Repetition interaction, $F(2,124)=$ $1.36, p=.26, M S_{\mathrm{e}}=0.415$, nor the Age $\times$ Rate interaction, $F(2,124)=0.073, p=.93, M S_{\mathrm{e}}=0.321$, was significant. A conservative estimate of power to detect a moderate size effect in these interactions at $p<.05$ was .71 (Cohen, 1988, Table 8.3.13, p. 314).

The conditional measure (percentage correct target report given recall of the pretarget) yielded comparable levels of recall for young and older subjects who recalled unrepeated targets ( $M=84.5 \%$ vs. $79 \%$ correct), but discarded more trials for older subjects $(13 \%)$ than for young subjects $(6 \%)$. The conditional data are shown in Figure 1 for young (left panel) and older (right panel) subjects. As can be seen in Figure 1, repeated targets were

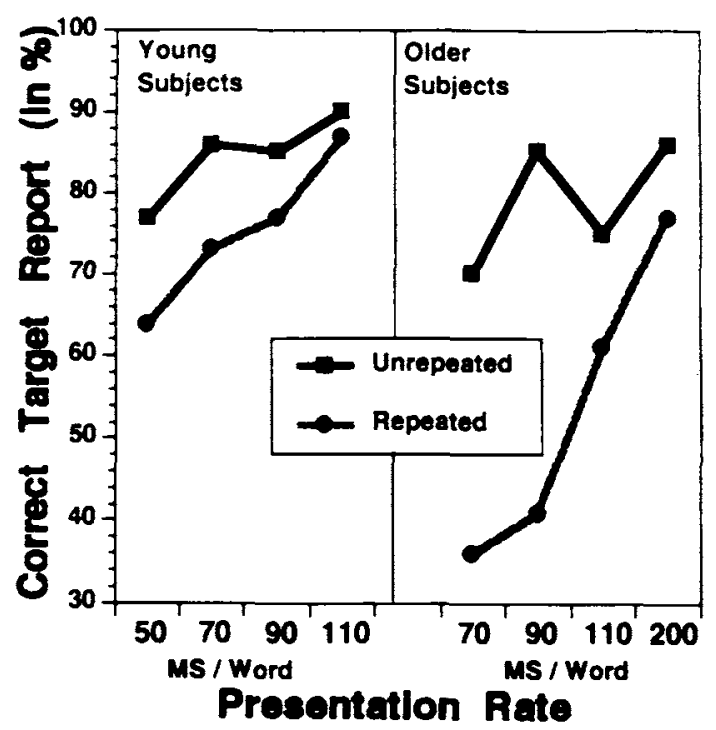

Figure 1. The conditional data: Correct target report (conditional, in percentages) for young subjects (left panel) and older subjects (right panel) as a function of repetition condition and rate (in milliseconds per word). reported less often than unrepeated targets across all four rates for young and older subjects. The Age $\times$ RB interaction was also apparent across all four rates, and recall of repeated targets did not appear either to deviate from linearity or to deviate from linearity at a faster rate for young than for older subjects. ANOVAs and trend analyses for the conditional data revealed identical statistical outcomes to those for the traditional measure and therefore corroborated conclusions from the traditional data.

\section{Discussion}

We first discuss our results in relation to methodological issues outlined in the introduction and to the inhibition deficit, refractory period, fusion, and binding hypotheses. We then develop a detailed theory within the binding framework that explains our results and generates new predictions for future testing.

\section{Methodologically Relevant Results}

As expected, use of nonidentical rates for young and older subjects helped rule out the possibility that differing effects of age on $\mathrm{RB}$ in the present study were attributable to ceiling effects. Considering only the identical rates $(70,90$, and $110 \mathrm{~ms}$ per word), conditional recall for unrepeated targets differed by more than $10 \%$ for young versus older subjects, which, taken alone, might raise the issue of whether our Age $\times$ Repetition interaction reflected effects of age on recall in the unrepeated control condition. The fact that RB diminished linearly with presentation time rather than just at the slowest rates is important for a similar reason: It cannot be explained as a ceiling artifact that is due to perfect recall of unrepeated targets at slower rates. Finally, by discarding more than twice as many trials for older subjects relative to young subjects, the conditional measure illustrated its weakness, which counteracts its benefits for statistical analyses of effects of aging on RB.

\section{$R B$ and the Inhibition Deficit Hypothesis}

The inhibition deficit hypothesis predicted less RB for older than for young adults, contrary to the present results (greater 
RB for older than young adults). Although RB is an inhibitory phenomenon at an empirical level, the present results suggest that the theoretical process underlying RB is not fundamentally inhibitory in nature. However, we cannot be sure that empirical inhibition deficits demonstrated in currently studied paradigms (e.g., Connelly et al., 1991, Hasher et al., 1991; Hasher \& Zacks, 1988; McDowd \& Fillion, 1992; Tipper \& Baylis, 1987; and Tipper, Borque, Anderson, Brehaut, \& Bastedo, 1990) apply to RB. Perhaps RB inhibition differs from empirical inhibition effects demonstrated in previous paradigms. Furthermore, even if $\mathrm{RB}$ involves the same type of inhibition, the present data only rule out inhibitory explanations of RB if future studies succeed in demonstrating age-linked inhibitory deficits at rapid presentation rates resembling those examined here.

Neither of the above caveats applies to theoretical inhibition as postulated in node structure theory (NST; MacKay, 1987, 1990; MacKay \& Burke, 1990). NST postulates an age-linked deficit in transmission of excitatory and inhibitory priming across connections between nodes, and this transmission deficit will occur for all processing rates and all inhibitory priming. Present data therefore rule out NST inhibition as the sole contributing factor in RB; however, it remains possible that both NST inhibition and binding play opposing but unequal roles in $\mathrm{RB}$, such that binding deficits offset inhibitory deficits in the data of older subjects. Another possibility is that the inhibition involved in RB increases in degree with age and experience (see MacKay, 1987, pp. 144-146), thereby offsetting the inhibitory deficits of older subjects.

\section{Refractory Period and Fusion Hypotheses}

The refractory period and fusion hypotheses predicted Age $\times$ Rate and Age $\times$ Rate $\times$ Repetition interactions, with older adults experiencing more $\mathrm{RB}$ than young adults at moderate rates but equivalent $\mathrm{RB}$ at fast rates and no $\mathrm{RB}$ for either group at slow rates. Present results for both overlapping rate analyses and identical rate analyses failed to support either of these predicted interactions. Moreover, confidence is warranted in the nonsignificance of these interactions: Because power of .80 is considered sufficient to detect a moderate-size interaction at $p$ $<.05$ (Cohen, 1988, p. 56), estimated power in our design was more than adequate to guard against Type II errors in the fourrate case $(.84)$. Even in the three-rate case, for which estimated power was .71 , actual power was probably greater than .80 , because power increases in degree with increased dependence among observations, and our estimates (based on Cohen, 1988, p. 316) did not take into account the fact that both Rate and Repetition were within-subject measures in our design.

The present results also failed to support the prediction, based on the fusion and refractory hypotheses, that RB would vary as a cubic function of age and presentation rate, with deviations from linearity in recall of repeated targets appearing at a faster rate for young than for older subjects. However, our data did support the background assumption or precondition of these hypotheses that recall of unrepeated targets varies with presentation time in the same way (linearly) for young and older adults. The present results therefore fail to support the possibility that RB is related to perceptual fusion or to refractory periods during which a second response to the same stimulus is slowed. In this regard, it is of interest that Hochhaus and Marohn's (1991, Experiment 4) results also failed to support perceptual fusion accounts of RB.

\section{The Binding Hypothesis}

According to the binding hypothesis, RB occurs because encoding repeated words requires two instances of connection formation involving one node, making repeated words more difficult to encode than unrepeated words under the time pressure of RSVP, especially for older adults, who in general require more time than young adults to form new connections (see, e.g., MacKay \& Burke, 1990). This general hypothesis predicted greater RB for older than for young adults, which was in fact observed. However, the binding framework requires further specification to explain why forming two connections from one node is so problematic, why RB diminishes linearly with presentation time, and why differences in RB between young and older subjects diminish systematically with presentation time. For example, it is not the case that a second connection from one node can be formed only after some fixed period of time, because $R B$ would not diminish linearly with presentation time under that particular binding hypothesis. An adequate account of $\mathrm{RB}$ within the binding framework must also explain why RB differs from its auditory analogue, discussed next.

\section{Differences Between $R B$ and Repetition Deafness (RD)}

The present work is directly comparable with MacKay and Miller's (1992) study of RD in young and older adults, in which virtually identical spoken sentences were computer compressed to rates resembling those in the present study, a procedure known as rapid auditory presentation (RAP). Subject characteristics were comparable in these two studies, as were levels of young adults' correct conditional target recall of RSVP versus RAP sentences ( $80 \%$ vs. $79 \%$, respectively) and older adults (66\% vs. $65 \%$, respectively). However, RD occurred for RAP word lists in MacKay and Miller (1992), but not for young or older subjects hearing sentences, unlike the present $R B$ data.

\section{Explaining RB Within NST}

NST (MacKay \& Miller, 1992, 1994) is a binding theory that explains in detail both the present results and the differences between RD and RB. Under NST, the main basis for RB in sentences is that a single node represents a lexical concept in longterm memory and must be connected with two phrase-level nodes if the lexical concept is repeated (MacKay \& Miller, 1992, 1994). For example, consider the nodes illustrated in Figure 2 for comprehension of the sentence "They wanted to play sports but sports were not allowed." The single lexical node for the repeated concept (sports) must quickly connect with two specific phrase nodes- "to play sports (verb phrase)" and "sports were not allowed (proposition)" (see Figure 2). Lexical nodes for unrepeated concepts (e.g., to, were, and allowed) become connected with only a single phrase node (see Figure 2 ). These one-to-one connections can be formed quickly and in parallel, whereas the one-to-many connections from repeated concepts to phrase nodes require more time because they must be formed in sequence (MacKay, 1990). Thus, a single node can connect 


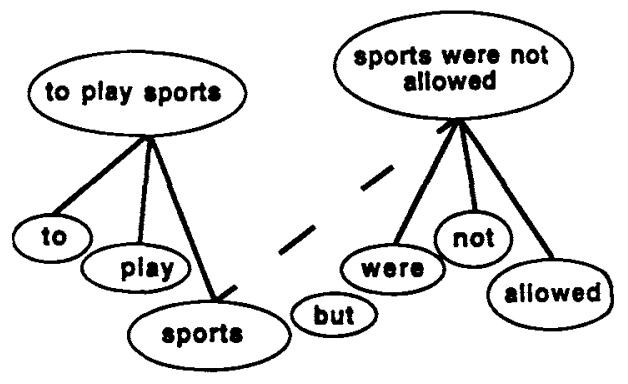

Figure 2. Selected nodes for encoding the sentence "They wanted to play sports, but sports were not allowed." Note that only a single lexical node represents the repeated word sports. The connection shown with a broken line is difficult to form under time pressure, the basis for repetition blindness.

with only one other node at a time, but no set period is required before a second connection can be formed. If the first connection from a repeated concept is formed, the second connection is less likely to be formed with short presentation times and few intervening words, causing RB, that is, failures to retrieve the second instance of the lexical concept and its phonology.

Therefore, graded factors that facilitate the formation of word-to-phrase links, for example, youth, or slower presentation rates, should cause systematic decreases in RB, as was observed. Another factor that can speed up the formation of wordto-phrase links is prosody, that is, acoustic cues to phrase boundaries such as timing, stress, pitch, and intonation (Levelt, 1990, pp. 365-412). To illustrate, consider how timing (pauses and word duration) helps listeners encode the acoustic sentence "Freud told me to go without hesitating." If $g o$ is lengthened and followed by a pause, as in "Freud instructed me to go-without hesitating," the listener can quickly link without hesitating to Freud's instruction. With reduced pausing and go length, however, listeners must link without hesitating to going. Such prosodic cues are present in RAP sentences but not in RAP lists or RSVP sentences, which may explain the differences between RB and RD. Normal prosody in RAP sentences will decrease RD by enabling listeners to quickly determine what words to link together into phrases, thereby increasing the time available for forming the one-to-many links required to encode and retrieve repeated words. However, RSVP imposes an unusual prosody in which each word in a sentence receives identical duration and identical $(0 \mathrm{~ms})$ between-word pauses and therefore may augment RB by slowing the formation of word-to-phrase links.

Differences between RD and RB also illustrate some ways in which NST differs from token individuation theory (Kanwisher, 1987; Kanwisher \& Potter, 1989). Token individuation theory treats lists the same as sentences and considers visual processing to be fundamentally different from auditory processing. Visually (but not auditorily) presented words are represented in two distinct ways in the brain, through a "type" node, which represents the item's identity, and through a "token" node, which represents each particular instance or occurrence of that item. According to token individuation theory, subjects recognize words in a list or sentence as types but cannot recognize the second instance of a repeated word as an individuated token, because of time limitations of RSVP. In principle, token nodes in token individuation theory must therefore outnumber word or type nodes in strings containing repeated words. Unlike type and token nodes, however, NST phrase nodes are always fewer in number than lexical or word nodes (see Figure 2) and do not constitute a fundamentally distinct means of representation. Phrase nodes represent different concepts from word (lexical) and phonological nodes (MacKay, 1987) but represent them in the same way: Representational character and basic processes do not differ for different nodes in NST. Nor can a phrase node be viewed as a token for a word type, whether repeated or not. For example, nodes representing the phrases as soon and as possible in "as soon as possible" do not constitute distinct tokens of $a s$, unlike the $a s^{1}-\mathrm{as}^{2}$ representations in token individuation theory. Phrase nodes simply represent different concepts from word nodes in NST, although an activated phrase node normally enables retrieval of connected words.

In conclusion, the present results replicate the RB phenomenon in young adults, extend it to older adults and to a parametric range of rates, and demonstrate that $\mathrm{RB}$ increases with age and decreases systematically with presentation time. These are new empirical phenomena that carry fundamental implications for the mechanisms underlying RB: RB is readily explained as a problem in encoding a single, theoretically specifiable connection rather than as a perceptual fusion, refractory period, or inhibitory process.

\section{References}

Banks, W. P., Burke, D. M., Krajicek, D., \& Whetstone, T. (1990). Psychology lab: Developing Macintosh courseware for introductory psychology classes. Unpublished manuscript, Pomona College, Claremont, CA.

Bavelier, D., \& Potter, M. (1992). Visual and phonological codes in repetition blindness. Journal of Experimental Psychology: Human Perception and Performance, 18, 134-147.

Birren, J. E., \& Woodruff, D. S. (1983). Aging: Past and future. In D. S. Woodruff \& J. E. Birren (Eds.), Aging: Scientific perspectives and social issues (2nd ed., pp. 1-15). Monterey, CA: Brooks/Cole.

Birren, J. E., Woods, A. M., \& Williams, M. V. (1980). Behavioral slowing with age: Causes, organization and consequences. In L. W. Poon (Ed.), Aging in the 1980's (pp. 293-308). Washington, DC: American Psychological Association.

Botwinick, J. (1984). Aging and behavior. New York: Springer.

Brown, R., \& McNeill, D. (1966). The "tip-of-the-tongue" phenomenon. Journal of Verbal Learning and Verbal Behavior, 5, 325-337.

Cohen, J. (1988). Statistical power analyses for the behavioral sciences (2nd ed.). Hillsdale, NJ: Erlbaum.

Connelly, S. L., Hasher, L., \& Zacks, R. T. (1991). Age and reading: The impact of distraction. Psychology and Aging, 6, 533-541.

Di Lollo, V., Arnett, J. L., \& Kruk, R. V. (1982). Age-related changes in the rate of visual information processing. Journal of Experimental Psychology: Human Perception and Performance, 8, 225-237.

Fine, E., \& Reeves, A. (1991, November). Variation of attention predicts repetition blindness in rapid serial visual presentation. Paper presented at the 32nd annual meeting of the Psychonomic Society, San Francisco.

Francis, W. N., \& Kucera, H. (1982). Frequency analysis of English usage: Lexicon and grammar. Boston: Houghton Mifflin.

Hasher, L., Stolfus, E. R., Zacks, R. T., \& Rympa, B. (1991). Age and inhibition. Journal of Experimental Psychology: Learning, Memory, and Cognition, 17, 163-169. 
Hasher, L., \& Zacks, R. T. (1988). Working memory and aging: A review and a new view. In G. H. Bower (Ed.), The psychology of learning and motivation (pp. 193-225). San Diego, CA: Academic Press.

Hochhaus, L., \& Marohn, K. M. (1991). Repetition blindness depends on perceptual capture and token individuation failure. Journal of $E x$ perimental Psychology: Human Perception and Performance, 17, $422-432$.

Humphreys, G. W., Besner, D., \& Quinlan, P. T. (1988). Event perception and the word repetition effect. Journal of Experimental Psychology: General, 117, 51-67.

Kanwisher, N. G. (1987). Repetition blindness: Type recognition without token individuation. Cognition, 27, 117-143.

Kanwisher, N. G. (1991). Repetition blindness and illusory conjunctions: Errors in binding visual types with visual tokens. Journal of Experimental Psychology: Human Perception and Performance, 17, 404-421.

Kanwisher, N. G., \& Potter, M. C. (1989). Repetition blindness: The effects of stimulus modality and spatial displacement. Memory \& Cognition, 17, 117-124.

Kanwisher, N. G., \& Potter, M. C. (1990). Repetition blindness: Levels of processing. Journal of Experimental Psychology: Human Perception and Performance, 16, 30-47.

Kline, D. W. (1984). Processing sense information. In J. Botwinick (Ed.), Aging and behavior: A comprehensive integration of research findings (pp. 207-228). New York: Springer.

Kline, D. W., Ikeda, D., \& Schieber, F. (1982). Age and temporal resolution in color vision: When do red and green make yellow? Journal of Gerontology, 37, 705-709.

Kline, D. W., \& Nestor, S. (1977). The persistence of complementary afterimages as a function of age and exposure duration. Experimental Aging Research, 3, 191-201.

Kline, D. W., \& Orme-Rogers, C. (1978). Examination of stimulus persistence as the basis for superior visual identification among older adults. Journal of Gerontology, 33, 76-81.

Levelt, W. J. M. (1990). Speaking: From intention to articulation. Cambridge, MA: MIT Press.

MacKay, D. G. (1969). The repeated letter effect in the misspellings of dysgraphics and normals. Perception and Psychophysics, 5, 102-106.

MacKay, D. G. (1987). The organization of perception and action: $A$ theory for language and other cognitive skills. New York: SpringerVerlag.

MacKay, D. G. (1990). Perception, action, and awareness: A three body problem. In W. Prinz \& O. Neumann (Eds.), Relationships between perception and action (pp. 269-303). Berlin, Germany: Springer-Verlag.

MacKay, D. G., \& Burke, D. M. (1990). Cognition and aging: A theory of new learning and the use of old connections. In T. Hess (Ed.), Aging and cognition: Knowledge organization and utilization (pp. 213-263). Amsterdam: North-Holland.

MacKay, D. G., \& Miller, M. (1992, April). The repetition deafness phenomenon in young and older adults. Paper presented at the 4th Biennial Cognitive Aging Conference, Atlanta, GA.

MacKay, D. G., \& Miller, M. (1994). Semantic blindness: Rapidly presented repeated concepts are difficult to encode and recall. Psychological Science, 5, 52-55.

McDowd, J. M., \& Fillion, D. L. (1992). Aging, selective attention, and inhibitory processes: A psychophysiological approach. Psychology and Aging, 7, 65-71.

McDowd, J. M., Oseas-Kreger, D. M., \& Filion, D. L. (1994). Inhibitory processes in selective attention and aging. In F. Demster (Ed.), New perspectives on interference and inhibition in cognition. San Diego, CA: Academic Press.

Tipper, S. P., \& Baylis, G. C. (1987). Individual differences in selective attention: The relation of priming and interference to cognitive failure. Personality and Individual Differences, 8, 667-675.

Tipper, S. P., Borque, T. A., Anderson, S. H., Brehaut, J. C., \& Bastedo, J. (1990). Mechanisms of attention: A developmental study. Journal of Experimental Child Psychology, 48, 353-378.

Wechsler, D. (1981). Manual for the Wechsler Adult Intelligence ScaleRevised. New York: Psychological Corporation.

Welford, A. T. (1977). Serial reaction times, continuity of task, singlechannel effects and age. In S. Dornic (Ed.), Attention and performance $V I$ (pp. 79-97). Hillsdale, NJ: Erlbaum.

Wickelgren, W. A. (1965). Short-term memory for repeated and nonrepeated items. Quarterly Journal of Experimental Psychology, 17, 14-25.

Received March 29, 1993

Revision received September 20, 1993

Accepted October 12, 1993 